Cita: Betancourt-Ocampo, D; Jaime-Reyes, A. L.; Tellez-Vasquez, M. H.; Rubio-Sosa, H. I.; González-González, A. (2022). Actividad física, sedentarismo y preferencias en la práctica deportiva en niños: panorama actual en México. Cuadernos de Psicología del Deporte, 22(1), $100-115$

\title{
Actividad física, sedentarismo y preferencias en la práctica deportiva en niños: panorama actual en México.
}

\section{Physical activity, sedentary lifestyle and preferences in sports practice in children: current panorama in Mexico.}

\section{Atividade física, sedentarismo e preferências na prática esportiva infantil: panorama atual no México.}

\author{
Betancourt-Ocampo, D. ${ }^{1}$, Jaimes-Reyes, A. L. ${ }^{1}$, Tellez-Vasquez, M. H. ${ }^{1}$, Rubio-Sosa, H. I. ${ }^{1}$, González- \\ González, A. ${ }^{1}$ \\ ${ }^{1}$ Universidad Anáhuac México
}

\begin{abstract}
RESUMEN
El presente estudio busco conocer la prevalencia de actividad física, sedentarismo y preferencias en la práctica deportiva en niños de México, por sexo y edad. Se seleccionó una muestra no probabilística de 2,621 niños (47.1\% niños y $52.5 \%$ niñas), con un promedio de edad de 9.86 años. Se utilizaron indicadores para evaluar la actividad física y las conductas sedentarias, así como, preguntas para conocer otros aspectos de las actividades físico deportivas. Los resultados mostraron que el mayor porcentaje de niños se clasifican como físicamente inactivos, encontrando diferencias significativas por sexo en el grupo de 11 a 13 años. Además, se encontró que una proporción importante de niños pasan más de dos horas al día frente a una pantalla. El mayor porcentaje de niños y niñas informaron que han practicado: fútbol soccer, correr y andar en bicicleta, y las actividades que más les gustaría practicar son el ping pong y escalar.
\end{abstract}

Palabras clave: Actividad física, conductas sedentarias, deportes, infancia.

\begin{abstract}
The present study sought to know the prevalence of physical activity, sedentary lifestyle and preferences in sports practice in children from Mexico, by sex and age. A non-probabilistic sample of 2,621 children (47.1\% boys and $52.5 \%$ girls) was selected, with an average age of 9.86 years. Indicators were used to evaluate physical activity and sedentary behaviors, as well as questions to learn about other aspects of physical sports activities. The results showed that the highest percentage of children are classified as physically inactive, finding significant differences by sex in the group aged 11 to 13 years. In addition, it was found that a significant proportion of children spend more than two hours a day in front of a screen. The highest percentage of boys and girls reported that they have practiced: soccer, running and cycling, and the activities they would most like to do are ping pong and climbing.
\end{abstract}

Keywords: Physical activation, sedentary behaviors, sports, childhood. 


\section{Betancourt-Ocampo et al.}

\section{RESUMO}

O presente estudo buscou conhecer a prevalência de atividade física, estilo de vida sedentário e preferências na prática esportiva em crianças mexicanas, por sexo e idade. Foi selecionada uma amostra não probabilística de 2.621 crianças (47,1\% meninos e 52,5\% meninas), com média de idade de 9,86 anos. Indicadores foram usados para avaliar a atividade física e comportamentos sedentários, bem como questões para aprender sobre outros aspectos das atividades físicas esportivas. Os resultados mostraram que o maior percentual de crianças é classificado como inativo fisicamente, encontrando diferenças significativas por sexo no grupo de 11 a 13 anos. Além disso, constatou-se que uma proporção significativa de crianças passa mais de duas horas por dia em frente a uma tela. O maior percentual de meninos e meninas relatou que já praticou: futebol, corrida e ciclismo, e as atividades que mais gostariam de fazer são pingue-pongue e escaladad

Palavras chave: Atividade física, comportamentos sedentários, esportes, infância.

\section{INTRODUCCIÓN}

En cuanto a actividad física diaria se refiere, el panorama mundial ha cambiado, lo que antes parecía normal y cotidiano, se ha vuelto excepcional; movernos, correr, saltar, divertirnos, resulta tan ajeno hoy en día, que pareciera ser que la inactividad física se ha incorporado como parte de nuestra naturaleza, lo cual no sólo resulta preocupante, sino que plantea uno de los retos más grandes de generaciones actuales: construir sociedades más sanas, caracterizadas por niños que corren, saltan y se divierten. Aunado a esto, son evidentes los costos que implica la inactividad física, en términos de salud y de obesidad infantil, además del impacto económico y a las consecuencias que esto representa, en los ámbitos: académico, familiar y psicológico; una sociedad que no se mueve detiene su crecimiento (Nike, 2016).

Pero, ¿cual es la importancia de una conducta que pareciera ser tan sencilla de llevar a cabo (la de moverse)? De acuerdo con algunas revisiones sistemáticas (Bailey, 2006; Eime et al., 2013; Owen et al., 2016; Poitras et al., 2016) son diversos los beneficios, tanto físicos como psicosociales, que obtienen tanto niños como adolescentes al realizar actividad física. Algunas de estas revisiones muestran que la actividad física se asocia con una mayor calidad de vida (Mollerup et al., 2017; Poitras et al., 2016; Rodríguez et al., 2020), con un menor riesgo de enfermedad (Bailey, 2006; Poitras et al., 2016), así como con niveles más altos en bienestar emocional y psicológico (Delgado-Floody et al., 2019; Eime et al., 2013; Li et al., 2018; Moral-Campillo et al., 2020; Poitras et al., 2016; Rodríguez et al., 2020), con un alto compromiso escolar (Owen et al., 2016), un buen rendimiento académico y funcionamiento cognitivo (Bekhechi \& Khiat, 2019; Domínguez et al., 2018; Suazo et al., 2019; Veraksa et al., 2021), mejores habilidades motoras (Piña et al., 2020; Poitras et al., 2016), comportamientos prosociales más frecuentes (Bailey, 2006). Además, las actitudes positivas sobre la actividad física a edades tempranas se consideran como un predictor de la actividad física durante la edad adulta (Bailey, 2006).

En el 2010, la Organización Mundial de la Salud (OMS) recomendó que para considerar a un niño como físicamente activo, éste debe realizar alrededor de 60 minutos al día de cualquier movimiento corporal que conlleve un consumo de energía, durante los siete días de la semana. Los niños pueden ir acumulando actividad física a lo largo del día mediante diferentes comportamientos, como el transporte a la escuela (por ejemplo, caminar o andar en bicicleta), el involucramiento en deportes o actividades organizadas y el juego al aire libre o dentro de los espacios del hogar. En el 2016, la OMS estimó que a nivel mundial sólo el $19,3 \%$ de los niños y adolescentes eran físicamente activos (World Health Organization, 2016a), lo cual, es un dato sumamente preocupante, ya que existe evidencia (e.g. Bailey, 2006; Pietilainen et al., 2008) que sugiere que los patrones de actividad física en los niños se asocian con los patrones de la etapa adulta, es decir, quienes no realizan actividad física en etapas tempranas del desarrollo tienen mayor probabilidad de tener un riesgo importante para presentar enfermedades crónicas no transmisibles como accidentes cerebrovasculares, diabetes y cáncer (Ortíz-Sánchez et 


\section{Preferencias en la práctica deportiva en niños mexicanos}

al., 2021; Soler-Lanagrán \& Castañeda-Vázquez, 2017).

Pero, ¿Qué ocurre a través del tiempo con la práctica de la actividad física y/o deportiva en los niños? Datos del estudio Health Behavior in School-aged Children [HBSC] el cual lleva realizándose ya por más de 30 años por la OMS y donde participan 49 países de Europa y América del Norte, reporta que el involucramiento en actividades físico deportivas van disminuyendo de la niñez a la adolescencia, específicamente se indica que de un $25 \%$ de los niños de 11 años que alcanzan el tiempo recomendado por la OMS, para los 15 años solo se ubica una proporción del $16 \%$, el informe indica una disminución significativa en la mayoría de los países de los cuales obtienen información (World Health Organization, 2016b). Además, se menciona que si bien, estos cambios se dan tanto para hombres como para mujeres, algunos hallazgos muestran que estas diferencias entre edades son más marcadas en mujeres que en hombres, así mismo, se menciona que el tipo de actividad de preferencia también es distinta por hombres y mujeres, donde a las adolescentes prefieren realizar actividades físico deportivas de menor intensidad y duración que los varones.

En este mismo sentido, Hernando et al. (2013) reportan diferencias en la actividad física y deporte en hombres y mujeres adolescentes (de entre 12 a 17 años de edad), donde los varones realizan con mayor frecuencia este tipo de actividades que las adolescentes; así mismo, al realizar análisis por grupo de edad y sexo encuentra datos consistentes por lo reportado por la OMS (World Health Organization, 2016b) respecto que a medida que aumenta la edad, va disminuyendo la frecuencia de actividad física y deporte, tanto en hombres como en mujeres, pero esta diferencia se da, de manera más clara en las mujeres que en los hombres.

Dada esta preocupante problemática, la OMS (2018) sugirió mantener un monitoreo a nivel mundial sobre los niveles de actividad física para tener datos que permitan valorar la efectividad de las políticas y programas que se realizan en cada país en la promoción de la activación física. Sin embargo, en México existe muy poca información sobre la prevalencia e incidencia de la actividad física en niños (International Society for Physical Activity and Health, 2016). De acuerdo con los datos de la Encuesta Nacional de Salud y Nutrición de México 2016
[ENSANUT] (Instituto Nacional de Salud Pública, Secretaría de Salud, 2016), el 51,1\% de los niños de entre 10 y 14 años de edad no realizan actividad física, $45,8 \%$ realizan de una a dos actividades y sólo el 3,1\% llevan a cabo de tres a más actividades; al realizar el análisis de acuerdo a los criterios que establece la OMS (2010) para determinar si una persona es activa o no, los datos del ENSANUT 2016 indicaron que la mayoría de los niños $(82,8 \%)$ se pueden categorizar como inactivos. Si bien, en el 2018 se aplicó la nuevamente la ENSANUT (Secretaría de Salud, Instituto Nacional de Salud Pública y Instituto Nacional de Estadística y Geografía, 2018), los hallazgos preliminares de esta encuesta reportan resultados de la población que es activa físicamente, pero solo se da información sobre población de 20 años o más.

Como se puede apreciar, la situación respecto a la activación física en población infantil en nuestro país es alarmante, lo cual, aunado a los datos que presenta la ENSANUT del 2018 en cuanto a que el 35,6\% de niños de entre 5 y 11 años en México sufren sobrepeso $(18,1 \%)$ y obesidad $(17,5 \%)$ vuelve más complicado el panorama (Secretaría de Salud, Instituto Nacional de Salud Pública y Instituto Nacional de Estadística y Geografía, 2018). Debido a que la actividad física es el único componente del gasto de energía que puede modificarse voluntariamente, debe considerarse como un elemento fundamental para incidir en el problema de peso poco saludable que tiene nuestra población infantil. Además, de acuerdo con datos del Observatorio Global de Obesidad (World Obesity Federation, 2019), México ocuparía el segundo lugar de obesidad infantil en la región de las Américas. Si bien, es complicado hacer comparaciones exactas entre los distintos países debido a que existen diferencias en algunos aspectos, tales como: años de recolección de información, rango de edad, entre otros; es innegable que nuestro país se ubica en los primeros lugares de obesidad infantil, lo que lo convierte en una de las prioridades para el sector salud y para nuestra sociedad en general.

Además de los datos sobre sobrepeso y obesidad, la ENSANUT (Instituto Nacional de Salud Pública, Secretaría de Salud, 2016) presenta información sobre indicadores de conductas sedentarias, específicamente evalúa el tiempo que pasan los niños de entre 10 y 14 años frente a una pantalla, el informe indica que 79\% de los niños y 75,6\% de las niñas pasaron más de dos 


\section{Betancourt-Ocampo et al.}

horas frente a una pantalla, contrario a lo que recomienda la American Academy of Pedriatrics (2016), quien sugiere que los niños no pasen más de horas frente una pantalla al día, ya que esto los pone en riesgo de padecer obesidad, así como problemas de adicción a este tipo de dispositivos y otro tipo de problemáticas, es por ello que es de suma importancia el regular el tiempo que pasan los menores frente a los dispositivos electrónicos.

Si aunado a este panorama, se considera que el gasto programable en cuestión de salud representó en nuestro país solo el 3,2\% del Producto Interno Bruto (PIB) en 2013, para el 2015 fue de 3,1\% y para el 2017 fue de $2,8 \%$, cifra muy por debajo a lo que estima la OCDE $(6.3 \%)$ que se necesita para proveer un sistema de salud óptimo (Dirección General de Información en Salud, 2019). En este mismo sentido, respecto a la inversión que se realiza en nuestro país en el ámbito deportivo, también se muestra una baja del presupuesto asignado en los últimos años, en el 2013 la Comisión Nacional de Cultura Física y Deporte (CONADE) recibió un monto de $\$ 7179$ millones de pesos, para el 2015 se le asignaron $\$ 3605$ millones de pesos y para el 2019 se le otorgaron $\$ 1718$ millones (Notimex, 2019); es importante mencionar, que para el 2020 en la propuesta del paquete económico (Cruz, 2019) para el deporte se contempla un presupuesto de $\$ 2,774,567,779$, lo cual sería un incremento substancial. Sin embargo, de acuerdo con Forteza (2020) dicho presupuesto no fue asignado en su totalidad debido a que el gobierno federal reasigno parte del presupuesto al área de salud, para el combate de la pandemia por COVID-19. Como podemos observar, el reto no es menor, dado que en México existe poca información sobre la actividad física y deportiva en población infantil, si bien, existen datos epidemiológicos sobre obesidad y sobrepeso desde edades muy tempranas, es escasa la información acerca de la actividad física y del tipo de actividades que practican los niños, así como de los motivos que tienen para practicar o no este tipo de actividades; es por ello, que el presente estudio tiene como objetivo conocer la prevalencia de actividad física, sedentarismo y preferencias en la práctica deportiva en niños de México, por sexo y edad.

\section{MATERIAL Y MÉTODOS}

\section{Diseño}

Se realizó un estudio no experimental, comparativo, con un diseño de cohortes transversal (Ato et al., 2013).

\section{Participantes}

Se seleccionó una muestra no probabilística de 2621 niños, $47.1 \%$ fueron niños y $52.5 \%$ niñas, de 13 localidades urbanas de 11 Estados de la República Mexicana (Baja California Norte, Coahuila, Nuevo León, Aguascalientes, Guanajuato, Jalisco, Michoacán, Estado de México, Puebla, Guerrero y Yucatán). Con un rango de edad de 8 a 13 años $(M=9.86, D E=1.24)$, quienes cursaban de tercero a sexto grado de educación primaria, la mayoría $(80,9 \%)$ informó vivir con ambos padres.

\section{Instrumentos}

Para evaluar la actividad física de los niños y las conductas sedentarias se utilizaron siete indicadores de la Encuesta Nacional de Salud y Nutrición 2016 (Instituto Nacional de Salud Pública, Secretaría de Salud, 2016). Uno de los indicadores evalúa el número de días en los que el niño realizó actividad física por al menos 60 minutos. Para las conductas sedentarias, se utilizaron seis indicadores que evalúan el tiempo promedio (en minutos) que pasa el niño frente a una pantalla en tres modalidades (TV, jugando videojuegos o utilizando computadoras, teléfonos o tableta electrónica) durante un día normal entre semana y en fin de semana.

Además, se utilizaron cinco preguntas del State of Play: Youth Sports Survey (The Aspen Institute, 2018), tres de las preguntas evalúan: las actividades físico deportivas que los niños han practicado alguna vez en la vida, las que han practicado en el último mes y las que les gustaría practicar, son preguntas cerradas donde se les dan 26 opciones de actividades deportivas (por ejemplo, fútbol soccer, básquetbol, béisbol, etc.) y los menores marcar tantas opciones como sea necesario. Las dos preguntas restantes que se tomaron de este instrumento fueron para obtener información tanto de las razones por las que los niños no practican deportes o bien no lo hacen de manera regular (con 15 opciones de respuesta) y los motivos del por qué les gusta practicar deportes (con 19 opciones de respuesta), ambas preguntas fueron cerradas y los niños podrían marcar tantas opciones como fuera necesario. 


\section{Preferencias en la práctica deportiva en niños mexicanos}

\section{Procedimiento}

El presente estudio se llevó a cabo dentro de los estándares requeridos por la Declaración de Helsinki, y cumpliendo con las Normas de Ética en la Investigación en Ciencias del Deporte y del Ejercicio (Harriss, MacSween, \& Atkinson, 2019); además, recibió la aprobación (Prot20212) del Servicio de Consultoría Ética de la Universidad a la que pertenecen los autores del estudio. Se solicitó permiso a las autoridades escolares para poder llevar a cabo la recolección de información con sus estudiantes. A los padres de familia se les envío información sobre el proyecto, donde se les explicó cuál era el objetivo y se les pidió que si estaban de acuerdo con que sus hijos participaran en el estudio firmaran el consentimiento informado. Sólo a los niños cuyos padres firmaron el consentimiento informado se les aplicó el instrumento de forma grupal, dentro de los salones de clase. La participación de los menores fue voluntaria y se les informó que podían dejar de responder los instrumentos en el momento que así lo quisieran. A los niños de $3^{\circ}$ y $4^{\circ}$ se les leyeron las preguntas y los menores iban marcando sus respuestas, a los niños de $5^{\circ}$ y $6^{\circ}$ se les leyeron las instrucciones y cada uno respondió el cuestionario de manera individual.

\section{RESULTADOS}

Los resultados mostraron que menos de una quinta parte de los participantes cumplen con la recomendación de actividad física de la OMS, es decir, que realicen actividad física los siete días de la semana por al menos 60 minutos al día (ver Tabla 1). Además, se puede observar que al hacer el comparativo respecto al grupo de edad, fue significativamente $\left(X^{2}=14.11\right.$, $\mathrm{p}=.001)$ mayor el porcentaje de niños de 8 a 10 años que cumplieron con el criterio que sugiere la OMS, respecto al grupo de 11 a 13 años. Otro aspecto que llamó la atención fue que en el grupo de niños de 11 a 13 años, fue significativamente mayor la proporción de niños que fueron físicamente activos que de niñas, lo cual no sucede con el grupo de niños de 8 a 10 años, donde se encontraron porcentajes similares entre niños y niñas.

\section{Tabla 1}

Prevalencia de actividad física por grupo de edad, sexo y total.

\begin{tabular}{lcccccc}
\hline & \multicolumn{2}{c}{$\begin{array}{c}\text { De 8 a 10 } \\
\text { años }\end{array}$} & \multicolumn{2}{c}{$\begin{array}{c}\text { De 11 a 13 } \\
\text { años }\end{array}$} & \multicolumn{2}{c}{ Total } \\
& \multicolumn{2}{c}{$(n=1689)$} & \multicolumn{2}{c}{$(n=917)$} & \multicolumn{2}{c}{$(n=2552)$} \\
\cline { 2 - 7 } & $\begin{array}{c}\text { Niños } \\
\%\end{array}$ & $\begin{array}{c}\text { Niñas } \\
\text { Niños }\end{array}$ & $\begin{array}{c}\text { Niñas } \\
\text { Niños }\end{array}$ & $\begin{array}{c}\text { Niñas } \\
\%\end{array}$ \\
\hline Activos & 19.4 & 19.6 & 16.3 & 11.4 & 18.3 & 16.5 \\
Inactivos & 80.6 & 80.4 & 83.7 & 88.6 & 81.7 & 83.5 \\
& & & & & & \\
\multicolumn{1}{c}{$\boldsymbol{X}^{2}$} & 0.01 & 4.60 & 1.37 \\
$\boldsymbol{p}$ & .923 & .032 & .242 \\
\hline
\end{tabular}

Otro aspecto del cual se obtuvo información fue el tiempo que pasan los niños frente a una pantalla (TV, jugando videojuegos o utilizando computadoras, teléfonos o tableta electrónica) en un día normal entre semana y en fin de semana, lo cual de acuerdo con la American Academy of Pedriatrics (2016) no tendría que exceder de dos horas al día, ya que esto se considera como un indicador de conductas sedentarias.

Tabla 2. Prevalencia de tiempo frente a una pantalla en un día entre semana por grupo de edad, sexo y total.

\begin{tabular}{rcccccc}
\hline & \multicolumn{2}{c}{$\begin{array}{c}\text { De 8 a 10 } \\
\text { años } \\
(n=1622)\end{array}$} & \multicolumn{2}{c}{$\begin{array}{c}\text { De 11 a 13 } \\
\text { años } \\
(n=893)\end{array}$} & \multicolumn{2}{c}{$\begin{array}{c}\text { Total } \\
(n=2526)\end{array}$} \\
\cline { 2 - 7 } & $\begin{array}{c}\text { Niños } \\
\%\end{array}$ & $\begin{array}{c}\text { Niñas } \\
\%\end{array}$ & $\begin{array}{c}\text { Niños } \\
\%\end{array}$ & $\begin{array}{c}\text { Niñas } \\
\%\end{array}$ & $\begin{array}{c}\text { Niños } \\
\%\end{array}$ & $\begin{array}{c}\text { Niñas } \\
\%\end{array}$ \\
\hline $\begin{array}{c}<\mathbf{2 h r} \\
\text { al día } \\
>\mathbf{2 h r}\end{array}$ & 12.6 & 12.6 & 8.8 & 10.7 & 11.2 & 12.0 \\
al día & 87.4 & 87.4 & 91.2 & 89.3 & 88.8 & 88.0 \\
& & & & & & \\
$\boldsymbol{X}^{2}$ & 0.00 & 0.94 & & 0.35 \\
$\boldsymbol{p}$ & .998 & .332 & & .552 \\
\hline
\end{tabular}

Los resultados respecto a un día entre semana (ver Tabla 2) mostraron que menos del $12 \%$ de los niños cumplen con la recomendación de estar hasta dos horas al día frente a una pantalla en un día entre semana, es decir, que cerca del $88 \%$ de los niños pasan más de dos horas frente a una pantalla. Al hacer el comparativo por grupo de edad, los resultados mostraron diferencias significativas $\left(X^{2}=4.17\right.$, $p=.041$ ), donde fue mayor el porcentaje de niños de 11 a 13 años que pasan más de dos horas frente a una pantalla respecto al grupo de 8 a 10 años. Así mismo, los resultados mostraron que no hubo diferencias significativas (ver Tabla 2) en las conductas sedentarias entre niños y niñas, específicamente en el grupo de 8 a 10 años fueron los mismos porcentajes para hombres y mujeres, y en el grupo de 11 a 13 años, fue ligeramente mayor el porcentaje de niñas que cumplen con la recomendación que de los niños, esto 


\section{Betancourt-Ocampo et al.}

es, que los niños tuvieron más conductas sedentarias que las niñas de este grupo de edad.

Por lo que respecta a los resultados sobre el tiempo que pasan los niños frente a una pantalla en un día en fin de semana, se encontraron diferencias significativas por grupo de edad $\left(X^{2}=6.23, p=.013\right)$, donde es mayor la proporción de niños de 11 a 13 años que pasan más de dos horas frente a una pantalla en contraste con los niños de 8 a 10 años. Al hacer los análisis por grupos de edad y por sexo, los hallazgos indicaron diferencias significativas sólo en el grupo de 8 a 10 años, donde a pesar de que la mayoría de niños y niñas pasan más de dos horas frente a una pantalla en un día promedio de fin de semana, es mayor el porcentaje de los varones que de mujeres, estos resultados se mantienen para el total de la muestra (Tabla 3). En el grupo de niños de 11 a 13 años, no se encontraron diferencias significativas por sexo.

Por otro lado, se les preguntó a los niños qué actividades físico deportivas habían practicado alguna vez en la vida, los hallazgos (ver Tabla 5) mostraron que para los niños de ambos grupos de edad, las

actividades que se mencionaron con mayor frecuencia fueron: fútbol soccer, andar en bicicleta y correr. Para
Tabla 3. Prevalencia de tiempo frente a una pantalla en un día en fin de semana por grupo de edad, sexo y total.

\begin{tabular}{|c|c|c|c|c|c|c|}
\hline & \multicolumn{2}{|c|}{$\begin{array}{c}\text { De } 8 \text { a } 10 \\
\text { años } \\
(n=1622)\end{array}$} & \multicolumn{2}{|c|}{$\begin{array}{c}\text { De } 11 \text { a } 13 \\
\text { años } \\
(n=893)\end{array}$} & \multicolumn{2}{|c|}{$\begin{array}{c}\text { Total } \\
(n=2526)\end{array}$} \\
\hline & $\begin{array}{c}\text { Niños } \\
\%\end{array}$ & $\begin{array}{c}\text { Niñas } \\
\%\end{array}$ & $\begin{array}{c}\text { Niños } \\
\%\end{array}$ & $\begin{array}{c}\text { Niñas } \\
\%\end{array}$ & $\begin{array}{c}\text { Niños } \\
\%\end{array}$ & $\begin{array}{c}\text { Niñas } \\
\%\end{array}$ \\
\hline \multirow{2}{*}{$\begin{array}{l}<2 h r \\
\text { al día } \\
>2 h r \\
\text { al día }\end{array}$} & 7.2 & 11.1 & 5.2 & 7.3 & 6.5 & 9.7 \\
\hline & 92.8 & 88.9 & 94.8 & 92.7 & 93.5 & 90.3 \\
\hline$X^{2}$ & \multicolumn{2}{|c|}{7.26} & \multicolumn{2}{|c|}{1.59} & \multicolumn{2}{|c|}{8.63} \\
\hline$p$ & \multicolumn{2}{|c|}{.007} & \multicolumn{2}{|c|}{.206} & \multicolumn{2}{|c|}{.003} \\
\hline
\end{tabular}

En cuanto a las razones que tienen los niños para no practicar o no hacerlo de manera regular actividades físico deportivas (ver Tabla 4). Los resultados mostraron que tener muchas responsabilidades y la falta de confianza en sus propias capacidades fueron las dos razones que se mencionaron por una mayor proporción de niños y niñas de ambos grupos de edad.

las niñas, correr, andar en bicicleta y caminar fueron las tres actividades reportadas con mayor frecuencia para ambos grupos de edad. mencionadas en ambos grupos de edad como, para el grupo de niñas de 8 a 10 años se encontró en tercer lugar bailar y para el grupo de 11 a 13 años el fútbol soccer

\begin{tabular}{|c|c|c|c|c|c|c|}
\hline & \multicolumn{2}{|c|}{$\begin{array}{c}\text { De } 8 \text { a } 10 \text { años } \\
\quad(n=1641)\end{array}$} & \multicolumn{2}{|c|}{$\begin{array}{c}\text { De } 11 \text { a } 13 \text { años } \\
(n=899)\end{array}$} & \multicolumn{2}{|c|}{$\begin{array}{c}\text { Total } \\
(n=2420)\end{array}$} \\
\hline & $\begin{array}{c}\text { Niños } \\
\%\end{array}$ & $\begin{array}{c}\text { Niñas } \\
\%\end{array}$ & $\begin{array}{c}\text { Niños } \\
\%\end{array}$ & $\begin{array}{c}\text { Niñas } \\
\%\end{array}$ & $\begin{array}{l}\text { Niños } \\
\%\end{array}$ & $\begin{array}{c}\text { Niñas } \\
\%\end{array}$ \\
\hline $\begin{array}{l}\text { Por tener muchas } \\
\text { responsabilidades }\end{array}$ & 41.2 & 43.5 & 43.5 & 41.4 & 42.1 & 42.8 \\
\hline Por aspectos del contexto & 12.0 & 17.0 & 11.7 & 13.9 & 11.8 & 15.9 \\
\hline Por factores económicos & 15.2 & 18.0 & 14.3 & 10.9 & 14.9 & 15.3 \\
\hline Por cuestiones de salud & 19.7 & 14.0 & 15.8 & 11.3 & 18.3 & 13.0 \\
\hline Por falta de confianza & 28.9 & 33.6 & 22.8 & 28.1 & 26.9 & 31.7 \\
\hline Por malas experiencias & 16.8 & 13.8 & 12.5 & 8.7 & 15.3 & 12.0 \\
\hline Falta de gusto o interés & 15.8 & 11.3 & 13.2 & 10.9 & 14.8 & 11.2 \\
\hline Faltan opciones & 14.9 & 15.3 & 13.2 & 12.9 & 14.4 & 14.4 \\
\hline Falta de posibilidades & 15.2 & 13.4 & 10.6 & 9.8 & 13.6 & 12.2 \\
\hline Falta de tiempo & 2.0 & 1.7 & 3.1 & 2.6 & 2.3 & 2.0 \\
\hline Falta de apoyo parental & 1.0 & 2.5 & 1.7 & 2.6 & 1.2 & 2.5 \\
\hline Uso inadecuado de tecnología & 0.2 & - & 0.7 & 0.4 & 0.4 & 0.1 \\
\hline
\end{tabular}




\section{Preferencias en la práctica deportiva en niños mexicanos}

Tabla 5. Prevalencia de actividades físico deportivas que han practicado los niños alguna vez en su vida, por grupo de edad, sexo y total.

\begin{tabular}{|c|c|c|c|c|c|c|}
\hline & \multicolumn{2}{|c|}{$\begin{array}{c}\text { De } 8 \text { a } 10 \text { años } \\
(n=1641)\end{array}$} & \multicolumn{2}{|c|}{$\begin{array}{c}\text { De } 11 \text { a } 13 \text { años } \\
(n=899)\end{array}$} & \multicolumn{2}{|c|}{$\begin{array}{c}\text { Total } \\
(n=2540)\end{array}$} \\
\hline & Niños & Niñas & Niños & Niñas & Niños & Niñas \\
\hline & $\%$ & $\%$ & $\%$ & $\%$ & $\%$ & $\%$ \\
\hline Básquetbol & 69.9 & 59.7 & 76.8 & 71.3 & 72.2 & 64.0 \\
\hline Fútbol soccer & 89.2 & 70.5 & 92.4 & 79.8 & 90.3 & 74.0 \\
\hline Fútbol americano & 38.7 & 14.5 & 47.0 & 17.6 & 41.5 & 15.6 \\
\hline Tochito bandera & 17.2 & 9.1 & 25.1 & 19.5 & 19.9 & 12.9 \\
\hline Béisbol & 40.2 & 22.6 & 43.0 & 25.2 & 58.9 & 41.1 \\
\hline Squash & 11.7 & 9.7 & 10.5 & 9.3 & 11.2 & 9.5 \\
\hline Andar en bicicleta & 74.5 & 76.2 & 78.0 & 81.0 & 75.5 & 78.0 \\
\hline Frisbee & 28.9 & 29.2 & 32.5 & 28.4 & 30.0 & 29.1 \\
\hline Voleibol & 52.1 & 49.1 & 58.2 & 66.6 & 54.1 & 55.4 \\
\hline Pelota pateada & 29.7 & 28.8 & 24.1 & 29.1 & 27.7 & 29.0 \\
\hline Natación & 49.8 & 56.5 & 53.7 & 59.3 & 51.0 & 57.5 \\
\hline Atletismo & 25.6 & 23.1 & 23.6 & 27.0 & 24.9 & 24.4 \\
\hline Gimnasia & 10.7 & 36.9 & 5.0 & 29.4 & 8.7 & 34.0 \\
\hline Badminton & 12.9 & 11.2 & 13.8 & 9.9 & 13.2 & 10.7 \\
\hline Animadora & 1.7 & 14.2 & 1.4 & 11.9 & 1.6 & 13.4 \\
\hline Boxeo & 30.4 & 13.0 & 29.9 & 11.8 & 30.3 & 12.5 \\
\hline Andar en patines & 31.9 & 68.8 & 31.8 & 65.7 & 31.7 & 67.7 \\
\hline Tenis & 25.6 & 19.9 & 27.9 & 20.9 & 26.4 & 20.1 \\
\hline Bailar & 41.5 & 75.4 & 48.6 & 74.9 & 43.8 & 75.0 \\
\hline Caminar & 69.4 & 76.1 & 77.3 & 88.5 & 72.0 & 80.6 \\
\hline Ping pong & 24.0 & 17.3 & 30.3 & 30.3 & 16.6 & 17.1 \\
\hline Andar en patineta & 31.9 & 21.4 & 33.2 & 17.2 & 32.3 & 19.9 \\
\hline Correr & 73.3 & 77.0 & 81.1 & 84.4 & 75.8 & 79.7 \\
\hline Escalar & 33.6 & 31.0 & 32.7 & 27.1 & 33.2 & 29.6 \\
\hline Karate o Taekwondo & 38.6 & 25.8 & 39.9 & 27.3 & 38.9 & 26.3 \\
\hline Frontón & 10.3 & 5.3 & 13.4 & 3.6 & 11.3 & 4.7 \\
\hline
\end{tabular}

En cuanto a las actividades físico-deportivas que han practicado los niños en el mes previo al estudio, los resultados indicaron que el fútbol soccer, correr y caminar fueron los más mencionados por los varones de ambos grupos de edad (Tabla 6). Para las niñas, caminar y correr, fueron las actividades más

Tabla 6.Prevalencia de actividades físico deportivas que han practicado los niños en el último mes, por grupo de edad, sexo y total.

\begin{tabular}{lcccccc}
\hline & \multicolumn{2}{c}{ De 8 a 10 años } & \multicolumn{2}{c}{ De 11 a 13 años } & \multicolumn{2}{c}{ Total } \\
& \multicolumn{2}{c}{$(n=1641)$} & \multicolumn{2}{c}{$(n=899)$} & \multicolumn{2}{c}{$(n=2540)$} \\
\cline { 2 - 7 } & Niños & Niñas & Niños & Niñas & Niños & Niñas \\
& $\%$ & $\%$ & $\%$ & $\%$ & $\%$ & $\%$ \\
\hline Básquetbol & 46.0 & 40.4 & 52.5 & 41.6 & 48.3 & 40.8 \\
Fútbol soccer & 76.6 & 50.1 & 78.2 & 59.3 & 77.2 & 53.2
\end{tabular}




\section{Betancourt-Ocampo et al.}

\begin{tabular}{lcccccc} 
Fútbol americano & 17.4 & 6.5 & 23.5 & 5.1 & 19.5 & 5.9 \\
Tochito bandera & 8.8 & 4.7 & 11.1 & 8.0 & 9.6 & 5.9 \\
Béisbol & 19.0 & 10.6 & 20.8 & 11.5 & 19.6 & 11.0 \\
Squash & 5.2 & 3.8 & 2.4 & 2.5 & 4.2 & 3.3 \\
Andar en bicicleta & 45.8 & 45.6 & 49.0 & 46.9 & 46.7 & 46.2 \\
Frisbee & 12.1 & 11.1 & 8.7 & 9.1 & 10.9 & 10.5 \\
Voleibol & 25.1 & 26.3 & 28.1 & 33.7 & 26.2 & 29.0 \\
Pelota pateada & 14.8 & 15.3 & 7.3 & 12.3 & 12.3 & 14.2 \\
Natación & 21.9 & 23.8 & 22.0 & 20.6 & 21.9 & 22.7 \\
Atletismo & 14.1 & 12.1 & 11.1 & 12.8 & 13.0 & 12.4 \\
Gimnasia & 4.9 & 18.1 & 1.7 & 11.7 & 3.8 & 15.7 \\
Badminton & 4.7 & 3.9 & 4.4 & 2.1 & 4.5 & 3.2 \\
Animadora & 1.8 & 7.2 & 0.7 & 4.3 & 1.4 & 6.2 \\
Boxeo & 16.2 & 7.6 & 14.8 & 6.0 & 15.9 & 6.9 \\
Andar en patines & 15.1 & 44.5 & 12.6 & 36.4 & 14.3 & 41.6 \\
Tenis & 10.6 & 7.4 & 7.3 & 7.6 & 9.6 & 7.4 \\
Bailar & 25.4 & 55.3 & 30.8 & 57.4 & 27.2 & 56.0 \\
Caminar & 52.1 & 61.0 & 65.6 & 74.3 & 56.8 & 65.8 \\
Ping pong & 9.2 & 6.7 & 9.0 & 4.7 & 9.1 & 6.0 \\
Andar en patineta & 18.1 & 10.6 & 18.4 & 6.6 & 18.1 & 9.1 \\
Correr & 54.2 & 61.4 & 70.0 & 73.4 & 59.6 & 65.8 \\
Escalar & 14.0 & 12.1 & 10.7 & 8.9 & 12.8 & 11.0 \\
Karate o Taekwondo & 16.8 & 10.7 & 11.9 & 6.2 & 15.1 & 9.0 \\
Frontón & 6.3 & 4.0 & 6.1 & 1.4 & 6.2 & 3.1 \\
\hline
\end{tabular}

En la Tabla 7 se presentan los hallazgos sobre las actividades físico deportivas que los niños y niñas mencionaron que les gustaría empezar a practicar, los resultados mostraron que para el grupo de niños de 8 a 10 años, en primer lugar se mencionó el ping pong, seguido del boxeo y en tercer lugar andar en patineta. Para los niños de 11 a 13 años, el boxeo y el ping pong fueron los deportes que se mencionaron con más frecuencia, seguidos de escalar y andar en patineta. Para las niñas, la gimnasia y ser animadora fueron las actividades más mencionadas en ambos grupos de edad, para las niñas de 8 a 10 años el atletismo se ubicó en tercer lugar de frecuencia y para las niñas de 11 a 13 años se ubicó el escalar.
Por otro lado, se les preguntó a los niños cuáles eran las razones por las que les gustaba practicar algún tipo de actividad físico deportiva. En el caso de los varones, las dos razones que mencionaron con mayor frecuencia fueron: jugar con sus amigos, divertirse y estar sano, esto para ambos grupos de edad (ver Tabla 8). Para las niñas, las razones con mayor frecuencia fueron: divertirse, estar sano y aprender cosas nuevas.

Tabla 7. Prevalencia de actividades físico deportivas que les gustaría practicar a los niños en el último mes, por grupo de edad, sexo y total.

\begin{tabular}{|c|c|c|c|c|c|c|}
\hline & \multicolumn{2}{|c|}{$\begin{array}{c}\text { De } 8 \text { a } 10 \text { años } \\
(n=1641)\end{array}$} & \multicolumn{2}{|c|}{$\begin{array}{c}\text { De } 11 \text { a } 13 \text { años } \\
(n=899)\end{array}$} & \multicolumn{2}{|c|}{$\begin{array}{c}\text { Total } \\
(n=2540)\end{array}$} \\
\hline & $\begin{array}{c}\text { Niños } \\
\%\end{array}$ & $\begin{array}{c}\text { Niñas } \\
\%\end{array}$ & $\begin{array}{c}\text { Niños } \\
\%\end{array}$ & $\begin{array}{c}\text { Niñas } \\
\%\end{array}$ & $\begin{array}{c}\text { Niños } \\
\%\end{array}$ & $\begin{array}{c}\text { Niñas } \\
\%\end{array}$ \\
\hline Básquetbol & 16.0 & 16.4 & 8.6 & 10.1 & 13.5 & 14.1 \\
\hline Fútbol soccer & 10.4 & 10.3 & 4.8 & 6.9 & 8.5 & 9.0 \\
\hline
\end{tabular}


Preferencias en la práctica deportiva en niños mexicanos

\begin{tabular}{|c|c|c|c|c|c|c|}
\hline Fútbol americano & 27.4 & 29.7 & 22.0 & 28.8 & 25.6 & 29.2 \\
\hline Tochito bandera & 28.3 & 32.3 & 27.5 & 23.7 & 27.9 & 29.1 \\
\hline Béisbol & 27.0 & 26.2 & 21.8 & 19.2 & 25.1 & 23.7 \\
\hline Squash & 29.1 & 29.7 & 32.9 & 25.8 & 30.2 & 28.2 \\
\hline Andar en bicicleta & 11.0 & 9.4 & 9.6 & 5.7 & 10.7 & 8.0 \\
\hline Frisbee & 24.4 & 24.9 & 24.9 & 18.0 & 24.4 & 22.4 \\
\hline Voleibol & 18.0 & 17.0 & 17.5 & 10.1 & 17.8 & 14.5 \\
\hline Pelota pateada & 21.5 & 19.0 & 21.1 & 15.0 & 21.2 & 17.6 \\
\hline Natación & 27.7 & 28.8 & 26.6 & 28.9 & 27.3 & 28.8 \\
\hline Atletismo & 37.0 & 40.3 & 34.0 & 35.6 & 35.8 & 38.5 \\
\hline Gimnasia & 28.4 & 46.1 & 29.5 & 53.0 & 28.7 & 48.6 \\
\hline Badminton & 29.1 & 29.1 & 32.9 & 23.5 & 30.3 & 27.2 \\
\hline Animadora & 12.5 & 49.8 & 12.7 & 48.6 & 12.6 & 49.4 \\
\hline Boxeo & 38.1 & 25.7 & 41.4 & 29.4 & 39.2 & 27.0 \\
\hline Andar en patines & 25.5 & 18.2 & 25.1 & 17.4 & 25.2 & 17.9 \\
\hline Tenis & 34.0 & 28.6 & 34.4 & 28.5 & 34.1 & 28.8 \\
\hline Bailar & 11.7 & 8.1 & 8.4 & 5.9 & 10.5 & 7.3 \\
\hline Caminar & 7.0 & 2.7 & 3.6 & 1.4 & 5.9 & 2.2 \\
\hline Ping pong & 39.1 & 39.0 & 41.4 & 35.1 & 39.6 & 37.5 \\
\hline Andar en patineta & 37.7 & 39.0 & 37.3 & 36.8 & 37.4 & 38.2 \\
\hline Correr & 9.6 & 4.7 & 5.8 & 3.4 & 8.3 & 4.2 \\
\hline Escalar & 29.3 & 36.9 & 37.6 & 39.9 & 31.9 & 38.0 \\
\hline Karate o Taekwondo & 35.1 & 40.2 & 35.6 & 39.1 & 35.2 & 39.9 \\
\hline Frontón & 30.5 & 32.9 & 33.7 & 27.7 & 31.5 & 31.0 \\
\hline
\end{tabular}

Tabla 8. Razones por las que les gusta a los niños practicar actividades fisico deportivas, por grupo de edad, sexo y total.

\begin{tabular}{|c|c|c|c|c|c|c|}
\hline & \multicolumn{2}{|c|}{$\begin{array}{c}\text { De } 8 \text { a } 10 \text { años } \\
(n=1570)\end{array}$} & \multicolumn{2}{|c|}{$\begin{array}{c}\text { De } 11 \text { a } 13 \text { años } \\
(n=841)\end{array}$} & \multicolumn{2}{|c|}{$\begin{array}{c}\text { Total } \\
(n=2423)\end{array}$} \\
\hline & $\begin{array}{c}\text { Niños } \\
\%\end{array}$ & $\begin{array}{c}\text { Niñas } \\
\%\end{array}$ & $\begin{array}{c}\text { Niños } \\
\%\end{array}$ & $\begin{array}{c}\text { Niñas } \\
\%\end{array}$ & $\begin{array}{c}\text { Niños } \\
\%\end{array}$ & $\begin{array}{c}\text { Niñas } \\
\%\end{array}$ \\
\hline Jugar con mis amigos & 67.9 & 60.5 & 79.0 & 60.0 & 71.5 & 60.4 \\
\hline Ejercitarme & 58.9 & 56.3 & 71.2 & 68.1 & 62.9 & 60.6 \\
\hline Hacer nuevos amigos & 51.0 & 43.8 & 56.1 & 42.7 & 52.4 & 43.4 \\
\hline $\begin{array}{l}\text { Para que mi familia se sienta } \\
\text { orgullosa de mí }\end{array}$ & 53.3 & 51.1 & 50.0 & 43.6 & 52.0 & 48.4 \\
\hline Aprender cosas nuevas & 58.5 & 66.0 & 67.4 & 73.9 & 61.4 & 69.0 \\
\hline Competir & 44.1 & 34.8 & 46.7 & 38.0 & 44.9 & 36.0 \\
\hline $\begin{array}{l}\text { Apoyar a mis compañeros de } \\
\text { equipo }\end{array}$ & 48.4 & 47.8 & 52.3 & 40.7 & 49.7 & 45.4 \\
\hline Jugar bien & 62.9 & 55.3 & 72.5 & 56.3 & 66.1 & 55.8 \\
\hline Ser el mejor en los deportes & 33.9 & 20.7 & 28.3 & 19.8 & 31.9 & 20.3 \\
\hline Jugar en un buen lugar & 41.1 & 39.6 & 37.9 & 33.3 & 40.0 & 37.3 \\
\hline Olvidarme de mis problemas & 39.9 & 38.1 & 49.5 & 45.2 & 43.1 & 40.7 \\
\hline Divertirme & 67.4 & 75.5 & 84.3 & 82.2 & 73.1 & 78.0 \\
\hline
\end{tabular}




\section{Betancourt-Ocampo et al.}

\begin{tabular}{lllllll} 
Tener un entrenador que se & 30.4 & 28.6 & 25.5 & 23.4 & 28.6 & 26.8 \\
$\begin{array}{l}\text { preocupa por mí } \\
\text { Tener nuevos uniformes }\end{array}$ & 27.7 & 16.8 & 14.9 & 12.1 & 23.2 & 15.2 \\
$\begin{array}{l}\text { Ganar } \\
\text { Sentir el apoyo de mis amigos } \\
\text { de equipo }\end{array}$ & 40.6 & 30.6 & 41.5 & 28.8 & 40.7 & 30.0 \\
$\begin{array}{l}\text { Desafiarme a mí mismo } \\
\text { Bajar de peso }\end{array}$ & 51.2 & 53.7 & 53.5 & 52.6 & 51.9 & 53.5 \\
Estar sano & 48.3 & 47.4 & 65.2 & 63.6 & 53.7 & 53.3 \\
\hline
\end{tabular}

También se les preguntó a los menores a qué edad empezaron a realizar actividades físico deportivas, y para determinar si había diferencia por sexo, se realizó una prueba $t$ de Student para muestras independientes. Los resultados mostraron diferencias significativas $(t$ $=-3.08, p<.010)$, donde las niñas presentaron un promedio de edad más alto $(M=5.34, D E=2.19)$, en comparación con los niños $(M=5.08, D E=1.92)$; no obstante, de acuerdo con los hallazgos, se puede apreciar que los menores iniciaron su activación deportiva aproximadamente a los cinco años.

Otro aspecto del cual se obtuvo información, fue sobre el lugar donde los menores suelen llevar a cabo actividades físico deportivas, como se puede observar en la Tabla 9, aproximadamente el $60 \%$ de los niños y niñas de ambos grupos de edad, mencionaron que lo realizan dentro del contexto escolar (e.g. dentro de la clase de educación física, recreo, etc.). En segundo lugar, se encontró, que los menores practican este tipo de actividades en deportivos, excepto las niñas de 8 a 10 años, quien en segundo lugar mencionaron el parque.

\section{DISCUSIÓN}

Hoy en día se conocen los distintos beneficios que tiene realizar actividad física de forma frecuente, los cuales, no solo enfocan en aspectos de salud física, sino también en aspectos psicológicos y sociales. Sin embargo, en México existe poca información sobre cuál es el panorama de la actividad física en población infantil, por lo que el presente estudio busco conocer tanto la prevalencia de actividad física, como del sedentarismo, así como las preferencias en la práctica deportiva en niños y niñas mexicanos.

Los datos tanto a nivel mundial (World Health Organization, 2016a) como de México (Instituto Nacional de Salud Pública, Secretaría de Salud, 2016), indican que menos de una quinta parte de niños y adolescentes que pueden considerarse como físicamente activos, lo cual, fue similar a lo encontrado en el presente estudio. Sin embargo, estos datos son preocupantes, ya que es muy baja la proporción de niños que cumplen con lo establecido por la OMS (2010) para poder ser catalogado como físicamente activo, lo cual se sabe conllevará costos importantes en diferentes ámbitos del desarrollo de los niños; además, de acuerdo con autores como Bailey (2006), el hecho de que los menores tengan actitudes favorables sobre la actividad física puede ser un buen predictor de que sigan practicando este tipo de actividades durante la edad adulta, lo cual podría contribuir a una disminución de enfermedades crónicas no transmisibles.

Tabla 9. Lugares donde suelen practicar actividades físico deportivas los niños, por grupo de edad, sexo y total.

\begin{tabular}{lcccccc}
\hline & \multicolumn{2}{c}{$\begin{array}{c}\text { De 8 a 10 años } \\
(n=1566)\end{array}$} & \multicolumn{2}{c}{$\begin{array}{c}\text { De 11 a 13 años } \\
(n=842)\end{array}$} & \multicolumn{2}{c}{$\begin{array}{c}\text { Total } \\
(n=2421)\end{array}$} \\
\cline { 2 - 7 } & Niños & Niñas & Niños & Niñas & Niños & Niñas \\
$\%$ & $\%$ & $\%$ & $\%$ & 68.0 & 63.2 & 61.9 \\
\hline Escuela & 59.8 & 58.4 & 69.8 & 47.6 & 54.9 & 43.2 \\
Deportivo & 45.3 & 41.0 & 48.6 & 46.4 & 41.9 & 42.4 \\
Parque & 41.8 & 45.6 & 41.8 & 3.9 & 6.0 & 8.0 \\
Casa & 5.9 & 8.2 & 6.3 & 7.0 & 1.9 & 2.4 \\
Calle & 1.5 & 2.9 & 2.5 & 1.5 & & \\
\hline
\end{tabular}




\section{Preferencias en la práctica deportiva en niños mexicanos}

Es importante señalar, que el hecho de que los niños no cumplan con los criterios establecidos por la OMS (2010), es decir, que un niño realice al menos 60 minutos al día (los siete días de la semana) de cualquier movimiento que conlleve consumo de energía para considerarse como físicamente activo, no implica que no realice actividad físico deportiva, más bien tiene que ver que no lo hace por el tiempo sugerido (siete días/60 minutos). Algo que tal vez debería considerarse, es la administración del tiempo que los niños tienen, ya que algunos autores (e.g. Téllez et al., 2020; Vignoli, 2019) sugieren que tanto los niños y/o adolescentes como sus padres mencionan que los menores no realizan actividades físicodeportivas debido a la falta de tiempo, esto debido a la carga de responsabilidades, tanto de los niños (actividades escolares, del hogar, etc.) como también la falta de tiempo de los padres, es por ello, que debe considerarse a la actividad física como una actividad primordial en el desarrollo del niño sobre otras actividades, lo cual favorecería que los menores realicen actividad física de manera más consistente, que a su vez, contribuirá de manera significativa a crear un buen hábito.

Otro aspecto que se encontró en la presente investigación, fueron las diferencias en cuanto a la actividad física entre niños y niñas, pero solo en el grupo de 11 a 13 años, donde fue mayor el porcentaje de niños que pudo catalogarse como físicamente activos en contraste con las niñas. Estos datos concuerdan con lo reportado por el estudio HBSC (World Health Organization, 2016b) y por el estudio de Hernando et al., (2013), donde además de reportar las diferencias por sexo, los autores explican que la actividad física tiene un punto crítico, al cambiar de la niñez a la adolescencia, es decir, este tipo de actividades drecrementan, en especial estos cambios se han aprecian más en mujeres. Si bien, en el presente estudio no se cuenta con adolescentes, el grupo de 11 a 13 años puede considerarse dentro de la pubertad, periodo del desarrollo donde se presentan cambios físicos y hormonales importantes (Craig \& Baucum, 2009), lo cual, podría estar asociado, a la baja de actividad, sobretodo en las mujeres, que es quienes regularmente inician antes toda esta serie de cambios.

De acuerdo con la ENSANUT (Instituto Nacional de Salud Pública, Secretaría de Salud, 2016), en México, existe una alta prevalencia de niños que pasan más de dos horas frente a una pantalla, lo cual, vale la pena subrayar se utiliza como indicador de conducta sedentaria; específicamente, los datos nacionales indican que $79 \%$ de los niños y $75,6 \%$ de las niñas (de entre 10 y 14 años) pasan más de horas frente a una pantalla. Los datos encontrados en el presente estudio apoyan los datos nacionales, en cuanto a que es mayor la proporción de menores que pasan más de dos horas frente a una pantalla respecto a los que pasan menos de dos horas; sin embargo, la prevalencia que se encontró fue aproximadamente 10 puntos porcentuales mayor, así mismo, algo que llamó la atención en el presente estudio es que al analizar la diferencia de este dato entre un día normal entre semana y un día de fin de semana, incrementa la proporción de niños y niñas que pasan más de horas frente a una pantalla en el fin de semana, lo cual, tal vez era de esperarse. Estos datos concuerdan con lo reportado por Guevara et al. (2019) donde en población adolescente también encontraron un mayor tiempo frente a la pantalla en los días de fines de semana respecto a un día normal entre semana. Además, no se puede dejar de lado que esto pudiera incrementar con la edad, ya que entre mayor edad, se esperaría que los menores pudieran contar con su propio teléfono móvil, lo que incrementa el tiempo frente a dispositivos electrónicos.

También es importante señalar que, al realizar el análisis por grupo de edad, los hallazgos del presente estudio mostraron que incrementa la proporción, tanto de niños como de niñas, que pasan más tiempo frente a una pantalla, si bien, ya se había mencionado que hay estudios (Hernando et al., 2013; World Health Organization, 2016b) que sugieren un cambio en el patrón de las conductas físico deportivas de la niñez a la adolescencia. Así mismo, es importante mencionar que el que los niños puedan categorizarse como activos físicamente no necesariamente implica que no realicen conductas sedentarias, al respecto, Perea et al. (2015) refieren que una persona podría presentar conductas sedentarias y ser activo físicamente o inactivo, no ser sedentario y activo físicamente, o bien, ser inactivo físicamente y no presentar conductas sedentarias. En este mismo sentido, Guevara et al. (2019) reportaron que no encontraron una asociación significativa entre el tiempo que pasaron un grupo de adolescentes frente a la pantalla con la actividad física que realizaban, lo cual, se comprueba en la presente investigación, donde el hecho de presentar más conductas sedentarias no necesariamente implicó que el niño no fuera activo físicamente, es decir, que los 


\section{Betancourt-Ocampo et al.}

niños pueden pasar muchas horas frente a la pantalla y a la vez realizar actividad física porque suceden en distintos momentos del día. No obstante, cuanto más tiempo pase el niño frente a una pantalla, es un tiempo en el que no está realizando actividad física, lo que implicaría que no está teniendo un gasto energético que pudiera contribuir a la prevención del sobrepeso, obesidad y enfermedades no transmisibles.

Uno de los objetivos primordiales del presente estudio fue conocer las preferencias en la práctica físico deportiva en niños y niñas, los hallazgos mostraron que los niños practican con mayor frecuencia el fútbol soccer, correr y andar en bicicleta, lo cual, en especial el fútbol soccer, podría ser algo que se esperaría ya que, en México es uno de los deportes más populares (Sánchez, 2018), junto con el boxeo, béisbol, básquetbol, lucha libre y futbol americano. Lo que llamó la atención, fueron los deportes que les gustaría intentar practicar a los niños y niñas, entre los más populares se encontraron: ping pong, escalar, boxeo (para los niños) y ser animadora (para las niñas), ya que no son de las prácticas deportivas más populares en el país. Estos datos sugieren que es importante generar y/o promover nuevas opciones para la práctica de actividad físico deportiva en México, y de este modo, ofrecerles una mayor variedad de opciones a los niños, ya que pudiera ser que si los niños perciben que no tienen mucha variedad fuera el motivo de la baja actividad física.

Por lo anterior, es primordial que se busque que los niños experimenten actividades físicas diferentes a las comunes y elijan aquellas con las que disfruten más; lo cual, podrá ayudar a que se conviertan en jóvenes y adultos físicamente activos. Lo que dejaría de lado la especialización deportiva temprana, que con el tiempo puede producir abandono, debido a los niveles de exigencia, al hartazgo o a la frustración que se experimenta al someterse a demandas cada vez mayores, no solo en lo físico, sino en cuestión de tiempos de dedicación y recursos económicos, entre otros aspectos (Mostafavifar et al., 2013).

Además de obtener la información sobre las preferencias de actividad físico deportiva, también se recabaron datos sobre las razones por las que a los niños les gusta practicarlas. En términos generales, los resultados mostraron que jugar con sus amigos, divertirse y estar sano, fueron las opciones más populares. Esta información es importante, ya que, si se parte del supuesto de que la mayoría de las ocasiones las actividades deportivas están organizadas por adultos, quienes frecuentemente buscan que los niños compitan y dejan a un lado la diversión, lo cual debería tener mayor prioridad ya que se considera un elemento fundamental en la iniciación deportiva. Al respecto, Visek et al. (2015) indican que al preguntar a los niños cuáles eran las principales razones que tenían para practicar deportes, nueve de cada diez niños respondieron que lo hacían solo por diversión, dejando de lado el ganar. Es por ello, que se debe tener en cuenta que son distintos los factores que ayudan a que el niño se divierta realizando actividad física y/o deportiva, entre ellos: el que sus padres les den la libertad para elegir lo que ellos quieren practicar, que los padres se sepan comportar si acuden con ellos a su práctica deportiva, además, un punto que les interesa a los niños es la aceptación de sus pares y de otros adultos, como puede ser el profesor de educación física y/o entrenador (Nike, 2016).

Un último aspecto que se analizó en el presente estudio fueron los lugares donde suelen realizar las actividades físico deportivas los niños, y la escuela, fue el espacio que se reporta con mayor frecuencia. Este dato es interesante, debido a que si bien no es el único espacio donde los menores pueden realizar este tipo de actividades, pareciera que se le está delegando esta responsabilidad al espacio educativo y donde, desafortunadamente hoy en día se sabe que al menos en México, los programas de educación física dentro del contexto escolar presentan una serie de limitaciones, una de ellas es que únicamente se destinan de 50 a 60 minutos a la semana para que los niños tengan actividad física y/o deportiva (Bonilla, 2016; Instituto Nacional de Salud Pública, 2018), lo cual es insuficiente, ya que, de acuerdo a la evidencia, en realidad solo son en promedio 37 minutos los que pueden considerarse como totalmente efectivos en actividad física (Bonilla, 2016). Aunado a la deficiencia de los programas educativos, también existen espacios educativos donde a la hora del descanso (receso) tampoco se les permite a los niños realizar actividad física por cuestiones de seguridad (e.g. caídas, accidentes, etc.), lo cual, limita a los menores y no les permite no realizar grandes movimientos y actividades, y que a su vez atenta contra su propio desarrollo.

\section{CONCLUSIONES}

Los resultados encontrados permiten concluir que un número importante de niños son inactivos físicamente, 


\section{Preferencias en la práctica deportiva en niños mexicanos}

lo cual, además se vincula con que suelen pasan un número importante de horas frente a las pantallas (e.g. computadora, teléfono, TV). Por lo que es importante, buscar estrategias que favorezcan que los niños incrementen el tiempo de actividad físico deportiva y tal vez, uno de éstos es moderar el número de tareas y responsabilidades que les dejan a los menores como parte de su formación académica, ya que fue le motivo que en el presente estudio se mencionó con mayor frecuencia. Además, es relevante buscar que los niños conozcan y practiquen diferentes actividades, para que encuentren a lo largo del tiempo, aquella que más les guste, de forma tal que puedan mantener la motivación para moverse y estar físicamente activos.

\section{APLICACIONES PRÁCTICAS}

Los datos obtenidos en el presente estudio podrían brindar información valiosa para la elaboración de políticas públicas en México, que promuevan en los niños el involucrarse con mayor frecuencia en actividades físico deportivas, lo cual, a su vez favorecerá el decremento de sobrepeso y obesidad infantojuvenil, así como las enfermedades asociadas a estas problemáticas. Las políticas deberían estar encaminadas a la alfabetización física y la promoción de los beneficios de practicar algún deporte, buscando otorgar a los niños una variedad de opciones para practicar y no buscar la especialización a corta edad.

Además, sería conveniente el promover actividades lúdicas, que permitan ver al deporte como una actividad libre y no sistemática, aburrida u obligatoria. Involucrar a los padres en las primeras experiencias deportivas de los niños que se realicen en los espacios educativos, haciendo énfasis en la diversión y convivencia más que en la competencia y descalificación. Generar campañas de concientización sobre la importancia de las primeras experiencias de los niños en actividades físico-deportivas, las cuales deben ser positivas y alentadoras, favoreciendo la cultura de la motivación más que la de los castigos. Promover juegos novedosos para los niños, dentro de los cuales pueden incluirse algunos juegos tradicionales, lo importante es enfocarse en el fomento del juego libre. Inspirar a los niños a través de adultos activos y saludables, apoyándose en la presencia de deportistas o atletas reconocidos en las actividades comunitarias. Sensibilizar a los profesores del papel que juegan como modelos de comportamiento ante sus alumnos, por lo que es importante que funcionen como modelos saludables.

\section{REFERENCIAS}

American Academy of Pediatrics. (2016). Media Use in School-Aged Children and Adolescents. Pediatrics, $138(5)$, e200162592. http://dx.doi.org/10.1542/peds.2016-2592.

Ato, M., López, J. J., \& Benavente, A. (2013). Un sistema de clasificación de los diseños de investigación en psicología. Anales de Psicología, 29(3), 1038-1059. doi: http://dx.doi.org/10.6018/analesps.29.3.178511

Bailey, R. (2006). Physical education and sport in schools: A review of benefits and outcomes. Journal of School Health, 76(8), 397-401. https://doi.org/10.1111/j.1746-1561.2006.00132.x

Bekhechi, A., \& K. Khiat, B. (2019) Impact of regular physical activity and sport on school performance among girls and boys aged between 6 and 10 years. Retos, 36, 398-402.

Bonilla, A. M. P. (2016). Impacto de la clase de educación física sobre la actividad moderada y vigorosa en niños de primaria. Revista Mexicana de Investigación en Cultura Física y Deporte, 1(1), 150173.

Craig, G. J., y Baucum, D. (2009). Desarrollo psicológico. México: Pearson Educación.

Cruz C. (8 de Septiembre de 2019). Plantean subir presupuesto a deporte a un año de Tokio 2020. Milenio. https://www.milenio.com/deportes/paqueteeconomico-2020-cual-es-el-presupuesto-para-eldeporte

Delgado-Floody, P., Jerez-Mayorga, D., CaamañoNavarrete, F., Carter-Thuillier, B., Cofré Lizama, A., \& Álvarez C. (2019). Psychological well-being related to screen time, physical activity after school, and weight status in Chilean schoolchildren. Nutrición Hospitalaria, 36(6), 1254-1260. doi: http://dx.doi.org/10.20960/nh.02751

Dirección General de Información en Salud. (13 de Mayo de 2019). Tabulados y Gráficos de Gastos en Salud. Secretaría de Salud: Gobierno de México. http://www.dgis.salud.gob.mx/contenidos/sinais/indic a_financieros_gobmx.html

Domínguez, G. F., Moral, C. L., Reigal, G. R. E., y Hernández, M. A. (2018). Condición física y atención 


\section{Betancourt-Ocampo et al.}

selectiva en una muestra preadolescente. Cuadernos de Psicología del Deporte, 18(2), 33-42.

Eime, R. M., Young, J. A., Harvey, J. T., Charity, M. J., \& Payne, W. R. (2013). A systematic review of the psychological and social benefits of participation in sport for children and adolescents: informing development of a conceptual model of health through sport. The International Journal of Behavioral Nutrition and Physical Activity, 10, 98. https://doi.org/10.1186/1479-5868-10-98

Forteza, H. (8 de septiembre de 2020). Designan presupuesto de 2 mil 700 mdp para Conade en 2021. El Heraldo de México. https://heraldodemexico.com.mx/deportes/2020/9/8/d esignan-presupuesto-de-mil-700-mdp-para-conadeen-2021-205506.html

Guevara, R. M., Urchaga, J. D., \& Sánchez-Moro, E. (2019). Horas de pantalla y actividad física de los estudiantes de Educación Secundaria. European Journal of Health Research, 5(2), 133-143. doi: 10.30552/ejhr.v5i2.184

Harriss, D. J., MacSween, A., \& Atkinson, G. (2019). Ethical Standards in Sport and Exercise Science Research: 2020 Update. International Journal of Sports Medicine, 40(13), 813-817. https://doi.org/10.1055/a-1015-3123

Hernando, Á., Oliva, A., y Pertegal, M. Á. (2013). Diferencias de género en los estilos de vida de los adolescentes. Psychosocial Intervention, 22(1), 15-23. https://doi.org/10.5093/in2013a3

Instituto Nacional de Salud Pública, y Secretaría de Salud. (2016). Encuesta Nacional de Salud y Nutrición de Medio Camino 2016. http://transparencia.insp.mx/2017/auditoriasinsp/12701_Resultados_Encuesta_ENSANUT_MC2 016.pdf

Instituto Nacional de Salud Pública. (2018). Hacia una estrategia nacional para la prestación de educación física de calidad en el nivel básico del sistema educativo Mexicano. México: Instituto Nacional de Salud Pública. Recuperado en https://unesdoc.unesco.org/ark:/48223/pf0000264037 .locale $=$ es

International Society for Physical Activity and Health. (2016). 1stPhysical Activity Almanac: The Global Observatory for Physical Activity-GoPA. https://indd.adobe.com/view/f8d2c921-4daf-4c969eaf-b8fb2c4de615

Li, Y. C., Graham, J.D., \& Cairney, J. (2018) Moderating Effects of Physical Activity and Global Self-Worth on Internalizing Problems in School-Aged Children With Developmental Coordination Disorder. Frontiers in Pshychology, 9, 1740. doi: 10.3389/fpsyg.2018.01740

Mollerup, P. M., Nielsen, T. R., Bøjsøe, C., Kloppenborg, J. T., Baker, J. L., \& Holm, J. C. (2017). Quality of life improves in children and adolescents during a community-based overweight and obesity treatment. Quality of Life Research, 26(6), 1597-1608. doi: 10.1007/s11136-017-1504-x

Moral-Campillo, L., Reigal-Garrido, R: E., \& Hernández-Mendo, A. (2020). Actividad física, funcionamiento cognitivo y psicosocial en una muestra preadolescente. Revista de Psicología del Deporte, 29(1), 123-132.

Mostafavifar, A. M., Best, T. M., y Myer, G. D. (2013). Early sport specialization, does it lead to longterm problems?. British Journal of Sports Medicine, 47(17), 1060-1061. http://dx.doi.org/10.1136/bjsports-2012-092005

Nike. (2016). Designed to move. A Physical Activity Action Agenda. https://www.nike.com/pdf/made-toplay-designed-to-move-2020-report.pdf

Notimex. (10 de abril de 2019). Presupuesto de Conade para 2019 rondará los mil 718 mdp. Publimetro.

https://www.publimetro.com.mx/mx/deportes/2019/0 4/10/presupuesto-conade-2019-rondara-los-mil-718mdp.html

Organización Mundial de la Salud. (2010). Recomendaciones mundiales sobre actividad física para la salud. https://apps.who.int/iris/bitstream/handle/10665/4444 1/9789243599977 spa.pdf;jsessionid=A9F4B1B296 60739A32B72697217EF5F3? sequence $=1$

Organización Mundial de la Salud. (23 de Febrero de 2018). Actividad física. https://www.who.int/es/newsroom/fact-sheets/detail/physical-activity

Ortíz-Sánchez, A., del Pozo-Cruz, J., Alfonso-Rosa, R. M., Gallardo-Gómez, D., \& Álvarez-Barbosa, F. (2021). Efectos del sedentarismo en niños en edad 


\section{Preferencias en la práctica deportiva en niños mexicanos}

escolar: revisión sistemática de estudios longitudinales. Retos, (40), 404-412.

Owen, K. B., Parker, P. D., Van Zanden, B., MacMillan, F., Astell-Burt, T., y Lonsdale, C. (2016). Physical activity and school engagement in youth: a systematic review and meta-analysis. Educational Psychologist, $\quad$ 51(2), 129-145. https://doi.org/10.1080/00461520.2016.1151793

Perea, S. J. M., Aparicio, V. A., Mascaraque, C. M., y Ortega, R. M. (2015). Actividad física y sedentarismo como moduladores de la situación nutricional. Nutrición Hospitalaria, 32(1),20-22.

Pietiläinen, K. H., Kaprio, J., Borg, P., Plasqui, G., Yki-Järvinen, H., Kujala, U. M., ... y Rissanen, A. (2008). Physical inactivity and obesity: a vicious circle. Obesity, 16(2), 409-414. https://doi.org/10.1038/oby.2007.72

Piña, D. D., Ochoa-Martínez, P. Y., Hall-López, J. A., \& Reyes, C. Z. E., Alarcón, M. E. I., Montreal, O. L. R., \& Sáenz-López, B. P. (2020). Efecto de un programa de educación física con intensidad moderada vigorosa sobre el desarrollo motor en niños de preescolar. Retos, 38, 363-368.

Poitras, V. J., Gray, C. E., Borghese, M. M., Carson, V., Chaput, J. P., Janssen, I., ... y Sampson, M. (2016). Systematic review of the relationships between objectively measured physical activity and health indicators in school-aged children and youth. Applied Physiology, Nutrition, and Metabolism, 41(6), S197S239. https://doi.org/10.1139/apnm-2015-0663

Rodríguez, R. J., Iglesias, F. A., \& Molina, L. J. (2020). Evaluación de la práctica de actividad física, la adherencia a la dieta y el comportamiento y su relación con la calidad de vida en estudiantes de educación primaria. Retos, 38, 129-136.

Sánchez, J. (5 de abril de 2018). Los seis deportes más populares en México. Sexenio. http://www.sexenio.com.mx/aplicaciones/articulo/def ault.aspx $? \mathrm{Id}=25834$

Secretaría de Salud, Instituto Nacional de Salud Pública, y Instituto Nacional de Estadística y Geografía. (2018). Encuesta Nacional de Salud y Nutrición 2018. Presentación de Resultados. https://ensanut.insp.mx/encuestas/ensanut2018/docto s/informes/ensanut_2018_presentacion_resultados.pd $\mathrm{f}$
Soler-Lanagrán, A., \& Castañeda-Vázquez, C. (2017). Estilo de vida sedentario y consecuencias en la salud de los niños. Una revisión sobre el estado en cuestión. Journal of Sport and Health Research, 9(2), 187-198.

Suazo, D. M., Muñoz, J. N., Lazarraga, P. C., Rodríguez, A. R., Alcayde, M. I., Roman, A. D., \& García, R. C. (2019). Mejora de la atención en niños y niñas con TDAH tras una intervención física deportiva dirigida. Cuadernos de Psicología del Deporte, 19(3), 37-46. https://doi.org/10.6018/cpd.360451

Téllez,V. M. H., Betancourt, O. D., Jaimes, R. A. L., Rubio, S. H. I., \& González-González, A. (2021). Motivos de inactividad física infantil: Una visión de niños, padres y entrenadores. Revista en Ciencias del Movimiento Humano y Salud, 18(2), 1-14. https://doi.org/10.15359/mhs.18-2.3

The Aspen Institute. (2018). State of Play: Youth Sports Survey. [Correo electrónico].

Veraksa, A., Tvardovskaya, A., Gavrilova, M., Yakupova, V., \& Musálek, M. (2021) Associations Between Executive Functions and Physical Fitness in Preschool Children. Frontiers in Psychology, 12, 674746. doi: 10.3389/fpsyg.2021.674746

Vignoli, H. (2019). Motivos de no realización de ejercicio físico $y / o$ actividad físico-deportiva en estudiantes de educación media superior (Tesis de Licenciatura, Instituto Universitario Asociación Cristiana de Jóvenes). http://accede.iuacj.edu.uy/bitstream/handle/20.500.12 729/221/TFG_2019_Vignoli_H.pdf?sequence=1\&is Allowed $=\mathrm{y}$

Visek, A. J., Achrati, S. M., Mannix, H. M., McDonnell, K., Harris, B. S., y DiPietro, L. (2015). The fun integration theory: toward sustaining children and adolescents sport participation. Journal of Physical Activity and Health, 12(3), 424-433. https://doi.org/10.1123/jpah.2013-0180

World Health Organization. (2016a). Global Health Observatory Data Repository. Prevalence of insufficient physical activity among school going adolescents. Geneva: WHO. http://apps.who.int/gho/data/view.main.2463ADO?la $\mathrm{ng}=\mathrm{en}$

World Health Organization. (2016b). Growing up unequal: gender and socioeconomic differences in young people's health and well-being. Health behaviour in school-aged children (HBSC) study: 


\section{Betancourt-Ocampo et al.}

International report from the 2013/2014 survey. http://www.euro.who.int/_data/assets/pdf_file/0003/ 303438/HSBC-No.7-Growing-up-unequal-FullReport.pdf?ua $=1$
World Obesity Federation. (2019). \% Sobrepeso infantil (incluida la obesidad) por región. https://pswod-webprod.s3.amazonaws.com/media/filer public/35/94/35 9430d5-bfd0-4ba6-ac1e-29d14ba3fb44/globces.pdf 